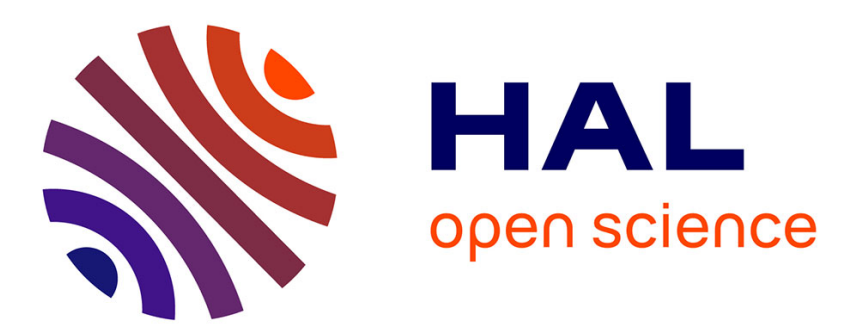

\title{
Exploring design space in embodiment design with consideration of models accuracy
}

\author{
Nicolas Perry, Mehdi El Amine, Jérôme Pailhes
}

\section{To cite this version:}

Nicolas Perry, Mehdi El Amine, Jérôme Pailhes. Exploring design space in embodiment design with consideration of models accuracy. CIRP Annals - Manufacturing Technology, 2015, 64 (1), pp.181-184. 10.1016/j.cirp.2015.04.021 . hal-01302644

\section{HAL Id: hal-01302644 \\ https://hal.science/hal-01302644}

Submitted on 14 Apr 2016

HAL is a multi-disciplinary open access archive for the deposit and dissemination of scientific research documents, whether they are published or not. The documents may come from teaching and research institutions in France or abroad, or from public or private research centers.
L'archive ouverte pluridisciplinaire HAL, est destinée au dépôt et à la diffusion de documents scientifiques de niveau recherche, publiés ou non, émanant des établissements d'enseignement et de recherche français ou étrangers, des laboratoires publics ou privés. 


\title{
Exploring design space in embodiment design with consideration of models accuracy
}

\author{
Nicolas Perry (2)*, Mehdi El Amine, Jérôme Pailhès \\ Arts et Métiers ParisTech, I2M - UMR 5295, F-33400 Talence, France
}

\begin{abstract}
A B S T R A C T
Rework tasks in collaborative development projects dealing with immature design concepts are very frequent and are responsible of cost overruns and schedule delays. Taking into account uncertainty and accuracy of models (and data) improves decision making according to strategic orientation for product development. Based on a real design of light, slender but rigid solar collector supports, multiple tools have been developed and tested to support decision making at different stages of development process. The objective of this paper is to investigate the integration of these accuracy evaluations into the design process. This proposal is validated by the industrial development and validation.
\end{abstract}

Keywords:

Decision making

Design

Concepts maturity

\section{Introduction and state of art}

Life-cycle cost can be influenced up to $70 \%$ by decisions taken during conceptual and embodiment design stages [1]. The need of supporting decision making by adequate theories and methodologies is thus greatest at these stages. For many years, design has been mostly dependent on companies' know-how and designers' tacit knowledge [2]. Nowadays, a great part of development time in design departments is spent on numerical calculation using behaviour models. Given the multitude hypothesis and approximations adopted in these models, their capacity to adequately represent product behaviours has always been a critical issue for designers. In addition, it is difficult to effectively qualify the accuracy of results provided by these behaviour models. This usually leads to a long "trial-and-error" process in which several physical prototypes are realized and tested. This is a frequent cause of cost overruns and schedule delays. Moreover, it provides no guarantee of approaching the optimal solution. Within this work, the objective is to propose a multi-criteria decision-making method that aims to maximize the satisfaction of design objectives with a consideration of the risk associated with the accuracy of behaviour models.

Multi-criteria decision making is a very complex problem in modern industry. As clearly explained by Krause and Golm [3] and Elwany et al. [4], it is principally due to the heterogeneous and contradictory nature of design objectives, and to data imprecision. In the framework of decision making in engineering design, the Method of Imprecision (MoI) proposed by Otto and Antonsson [5] has demonstrated its efficiency. It allows designers to formalize imprecision in Design Alternative (DA) evaluation using fuzzy logic. Each DA is then evaluated using several preference values

\footnotetext{
* Corresponding author.

E-mail address: nicolas.perry@ensam.eu (N. Perry).
}

which are finally aggregated into a generalized preference. In addition, the Mol proposes axioms to provide a framework for application to the field of engineering design, and the ability to use different strategies to express the will of designers. MoI provides solid theoretical basis in the field of decision making in engineering design. However, the main limit of this approach, in our context and for our point of view, is that the evaluation of DA does not take into account the accuracy degree of behaviour models (or data) used for the evaluation of DA. As an example, two DA may illicit the same performance in term of achievement of design goals but the risk related to the accuracy of behaviour models may be different. The attitude of Decision Maker (DM) in this case will not be the same.

Inaccuracy of behaviours models is due to: simplifying hypotheses, uncertainty in the identification of model parameters and, inaccuracy of digital resolution [6]. Evaluating the accuracy of a behaviour model is a difficult task. In the absence of significant background data, statistical tools such a case-based reasoning or artificial neural network cannot be used. In the present context, we suppose that reference points exist and correspond to results of experimental tests on real scale 1 prototype. In this case, it is recommended to measure accuracy as the distance between behaviours model result and reference points [7,8]. According to Vernat et al. [7], the accuracy estimation criteria can be local or global criteria. A local error estimate can be given, for example, through the maximum (Eq. (1)) or minimum of the absolute error in the measured points, or simply by the error at a particular point. A global error estimate can be given for example by the squared error (Eq. (2)) allows for example to obtain a general trend of the error on the entire design space. It is recommended to compare the obtained distance with a threshold value or accuracy objective $[7,8]$.

$E_{M}=\max _{i}\left|\tilde{X}_{i}-X_{i}\right|$ 
$\bar{E}=\sum_{i=1}^{n} \frac{1}{n}\left|\tilde{X}_{i}-X_{i}\right|$

The limit of such a measure of accuracy occurs when there are very few reference points and a large design alternatives space to explore. In this case, it is very difficult to justify the validity of this accuracy measure in all design alternatives space.

\section{Proposed approach}

When exploring the set of possible design alternatives using the results of behaviour models, decision maker's preferences are driven by two main considerations: (i) maximizing the satisfaction of design objectives, and (ii) minimizing the risk associated to the inaccuracy of behaviour models used. In the proposed approach, these two considerations are represented by numerical indicators that are respectively Overall Desirability Indicator (ODI) and, Safety Indicator (SI).

\subsection{Overall desirability indicator (ODI)}

ODI quantify the general level of satisfaction achieved by a DA. The model used for its calculation (Fig. 1) is similar to MoI [5]. Starting from a given DA that is defined by the choice of design parameters (structural dimensions, materials, etc.), behaviours models are used to determine the set of quantities needed to assess the achievement of design objectives (mass, maximum stress, carbon footprint, etc.). The resulting quantities are called Observation Variables $(\mathrm{OV})$. For each $\mathrm{OV}$, a Desirability Index $(D I)$ between 0 and 1 is then affected to express the level of satisfaction of the corresponding design objective (Fig. 2). In our approach, this process is made using the adjustable desirability function proposed by Harrington's [9] because of its ease of parametrization and its appropriateness to the different kinds of design objectives. The ODI is obtained by the aggregation of DI of each design objectives. The aggregation function used is Generalized Ordered Weighted Averaging (GOWA) operator (Eq. (4)) proposed by Yager [10]. This function was chosen since the aggregation strategy is completely adjustable by the choice of: (i) design objectives weights $\mathbf{w}_{\mathbf{i}}$ that express their relatives importance and, (ii) the parameter $\mathbf{s}$ that reflects the level of compensation between design objectives. Scott [11] proves that any DA in Pareto front can be reached by adjusting $\mathbf{w}_{\mathbf{i}}$ and $\mathbf{s}$. The interpretation of the given weights is not absolute but depends on the level of compensation (expressed by $\mathbf{s}$ ). In other way, the method used for the determination of weight must take into account the value of s. In order to respect this constraint, we use in this work the method of indifference points [11] that is based on the definition of indifference design alternatives that elicit the same

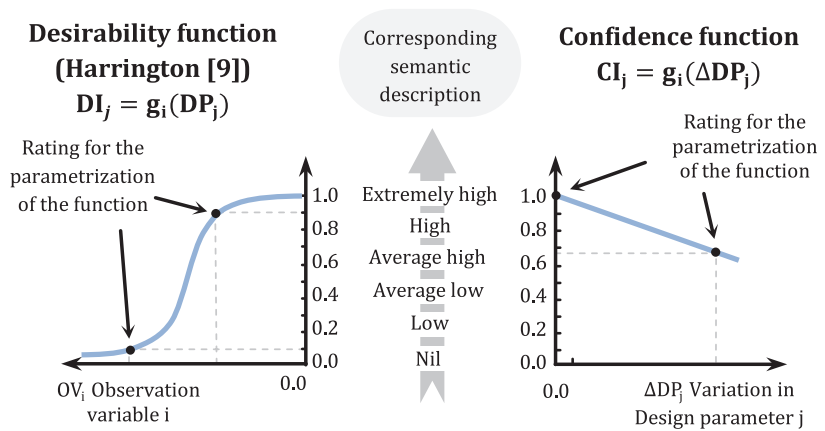

Fig. 2. Desirability and confidence functions.

performance, to determine simultaneously a unique value for the trade-off strategy and for the weight ratio.

$O D I=\sqrt[s]{\sum_{j}^{n}\left(w_{j}\left(D I_{j}\right)^{s}\right)}$

The step of defining desirability functions and aggregation function constitute a mean for capitalizing on knowledge associated with decision making. It also allows the automation the calculation process via the formalization of decision maker preferences. This facilitates the treatment of several DA. Another advantage of aggregation process is that it represents a mean of negotiating different and usually conflicting design objectives, which facilitate information exchange, mutual understanding, and joint decision-making in collaborative development [12].

\subsection{Safety indicator (SI)}

A recognized principle in engineering design decisions is that of annihilation $[5,13,14]$. It states that a minimum threshold is required for each design objective, and if also one of these thresholds is unmet, the DA is rejected regardless of the satisfaction level of the other design objectives. So, one of the main concerns of decision makers is to ensure the satisfaction of these minimum thresholds. The proposed Safety Indicator (SI) aims to assess the risk of not meeting the corresponding acceptance threshold due to the inaccuracy of behaviour models used. Given a design alternative, a Safety Indicator (SI) is calculated for each design objective and its value is to be maximized. In order to be conservative, the minimum value is considered between the different SI. The procedure to calculate SI is inspired from FMEA (Failure Mode Effects Analysis). Three factors are taken into account: the occurrence of the risk $(O)$, the gravity of not satisfying the acceptance threshold $(G)$ and, the detectability $(C)$. As the classic FMEA, gravity is estimated using a numerical scale (from

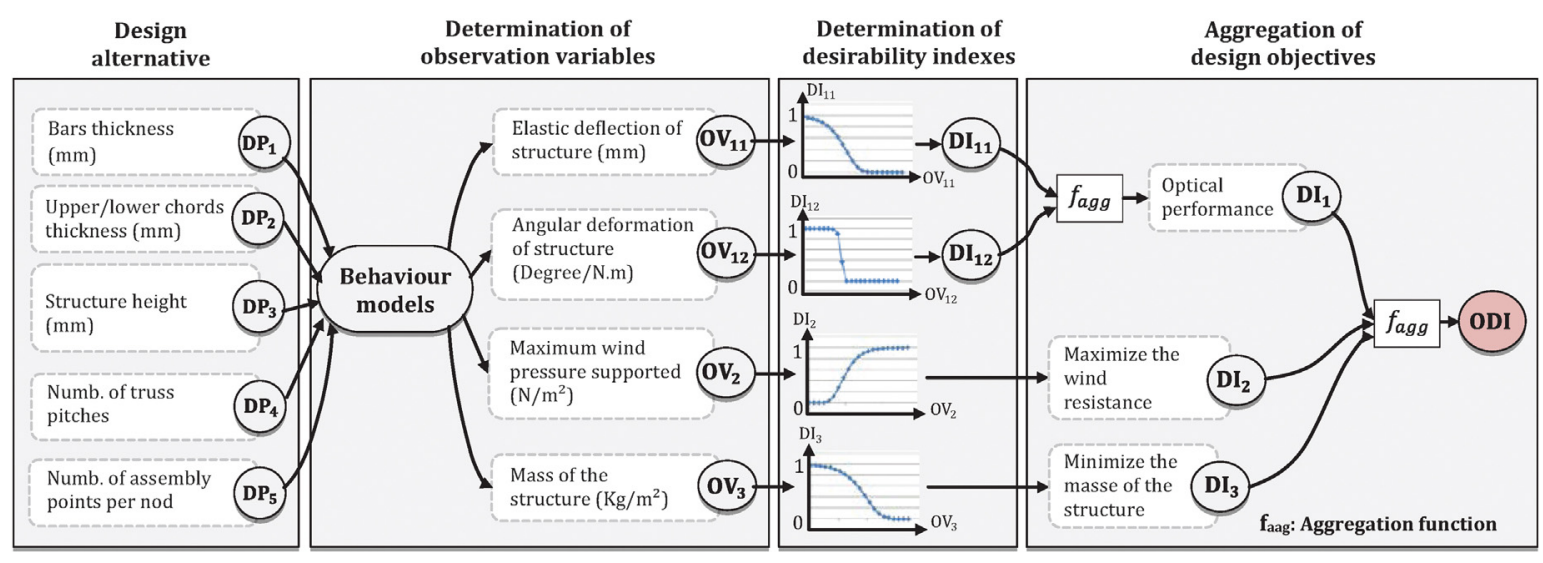

Fig. 1. Evaluation model of Overall Desirability Indicator. 
0.2 to 1 ) associated with semantic expressions. The methods of calculation of $O$ and $C$ are explained below. $S I$ is calculated by the relation:

$\mathrm{SI}=O \times G \times D$

\subsubsection{Occurrence of the risk $(\mathrm{O})$}

This factor is obtained by the relative distance between Observation Variables $(\mathrm{OV})$ calculated by behaviour model and the acceptance threshold. It is considered that the greater this distance is, the lower is the risk of not respecting acceptance threshold due to the low accuracy of behaviour model. In order to take into account the vagueness of acceptance threshold, a mean distance was calculated using two values $O V_{t}^{1}$ and $O V_{t}^{2}$ (Eq. (4)).

$O=\frac{1}{2} \times\left(D\left(O V_{m^{\prime}} O V_{t}^{1}\right)+D\left(O V_{m^{\prime}} O V_{t}^{2}\right)\right)$

where $D$ is the distance defined by:

$D(a, b)=\left\{\begin{array}{lll}\frac{|a-b|}{b} & \text { if } & a>b \\ 0 & \text { if } & a<b\end{array}\right.$

\subsubsection{Detectability (D)}

In our case, it is assumed that at least one design alternative has been prototyped and tested. It is considered as a reference point to evaluate the accuracy of behaviour model. For a given observation variable, the accuracy of the model is given by the distance between the value given by behaviour model $\left(O V_{i}\right)$ and the value given by experimental measures on physical prototype $\tilde{O} \tilde{V}_{i}$. The distance used is:

$D^{\prime}\left(O V_{i}, \tilde{O} \tilde{V}_{i}\right)=\left|\frac{O V-\tilde{O} \tilde{V}_{i}}{\tilde{O} \tilde{V}_{i}}\right|$

The conditions in which experimental tests on prototype was performed may be different of real environment. Moreover, measuring instruments always exhibit a certain imprecision. Experimental measures $\tilde{O} \tilde{V}_{i}$ are thus subject to imprecision. Assuming that experimental measure is comprised between $\tilde{O} \tilde{V}_{i}^{1}$ and $\tilde{O} \tilde{V}_{i}^{2}$, we consider an average distance $E$ obtained by the following equations:

$E=\left\{\begin{array}{lll}1-\frac{\left(D^{\prime}\left(O V_{i}, \tilde{O} \tilde{V}_{i}\right)\right)}{D_{s}^{\prime}} & \text { if } & D^{\prime}\left(\tilde{O} \tilde{V}_{i}, O V_{i}\right)<D_{s}^{\prime} \\ 0 & \text { if } & D^{\prime}\left(\tilde{O} \tilde{V}_{i}, O V_{i}\right)>D_{s}^{\prime}\end{array}\right.$

$\left\langle D^{\prime}\left(O V_{i}, \tilde{O} \tilde{V}_{i}\right)\right\rangle=\frac{D^{\prime}\left(O V_{i}, \tilde{O} \tilde{V}_{i}^{1}\right)+D^{\prime}\left(O V_{i}, \tilde{O} \tilde{V}_{i}^{2}\right)}{2}$

$D_{s}^{\prime}$ is a threshold distance over which the model is considered unusable by the designer. In our case study, we adopt a value of 0.20 for each observation variable.

The distance $E$ is calculated for the design alternative corresponding to the prototype. For a different design alternative, in which different design parameters are used, this accuracy measure is no longer valid. In order to correct this indicator, it is multiplied by a second term (Eq. (9)) that represents the degradation of behaviour model exactness related to the distance of the evaluated design alternative from the physical prototype.

$D=E \times \prod_{i}^{n} C D_{i}=\prod_{i}^{n} g_{i}\left(D P_{i}-D P_{o}\right)$

$n$ is the number of design parameters and $C_{i}$ is a parameter comprised between 0 and 1 and express the degradation of measured accuracy $E$. It is calculated using confidence functions $g_{i}$ (Fig. 2) that express $C D_{i}$ as a function of the distance between the design parameter of the reference solution $C D_{o}$ and that of candidate solution $C D_{i}$. As shown in Fig. 2, this function is parametrized using two points that are estimated by designers involved in the development of the product. This additional information allows extending the use of initial accuracy assessment to all design alternatives space.

\section{Application to the industrial case}

In a concentrated solar power (CSP) plant, the main function of solar collectors is to concentrate and redirect sunlight onto absorber tubes to heat up the working fluid. The solar collector is composed of reflective plates (reflective glasses plates or polished aluminium plates) and a supporting structure whose function is to give and maintain reflective plates shape (Fig. 3). A mounting device is placed between the reflecting surface plates and the supporting structure to ensure the connection between them. In our study, only supporting structure is considered. The concept selected in conceptual design phase is truss structure. The cost of raw material for the manufacturing of solar collectors represents $50 \%$ of investment cost for a CSP plant [15]. It is thus important to reduce the structure mass (objective $\mathrm{DI}_{3}$ ). In addition, in order to maintain a good optical performance, elastic deformation of the structure must remain as low as possible (objective $D I_{1}$ ). Finally, it must resist a high wind pressure to be usable for a multitude of implementation sites (objective $\mathrm{DI}_{2}$ ). More details about the product are given in [14].

A first behaviour model based on bars-type finite element analysis has been proposed to evaluate $O V$. Using this model, a design of experiment of design parameters has been performed and a first DA has been selected for prototyping (Fig. 3). The comparison between mechanical tests and behaviours model $\mathrm{OV}$ reveals major discrepancies (until 35\% of error). The first behaviour model is not adapted to this structure since it does not correctly reproduce physique phenomena in nods connexions, which are strongly involved in the evaluation observation variables. A second behaviour model has been proposed using shell-type finite element analysis with a highly refined meshing in nods connexions. The prototype testing results were used as a reference point to test the accuracy of the improved behaviour model and a second design of experiment procedure has been performed by varying design parameters in the established ranges. Each of DA has been tested in term of ODI and SI. Fig. 4 shows the results of design of experiment.

At this stage of development, decisions are required on the evolution of actual DA (prototyped alternative) and on the
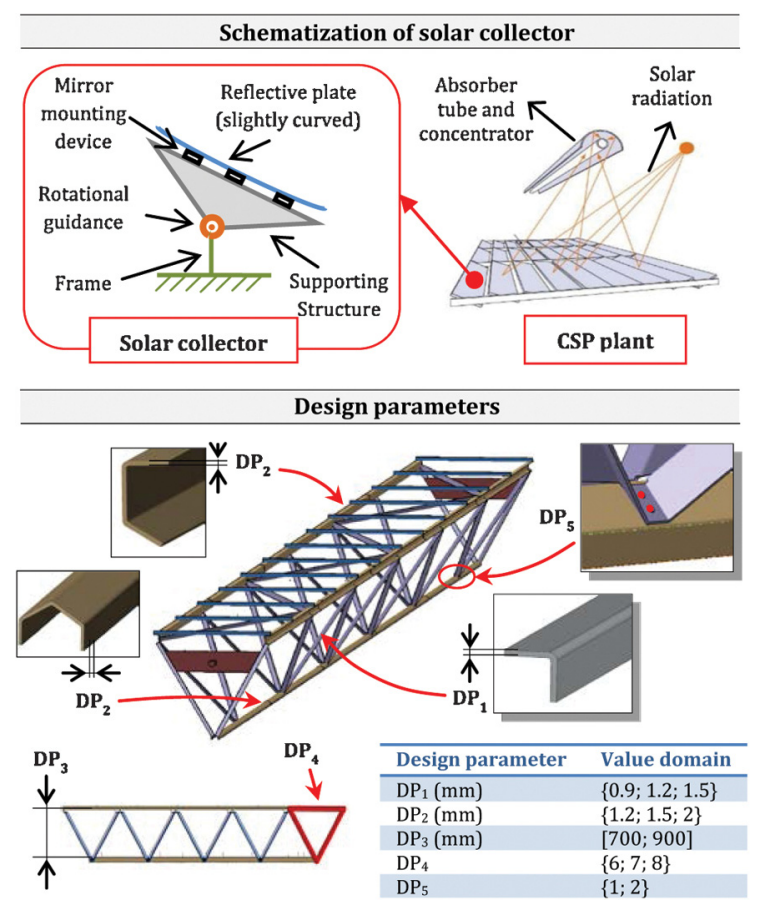

Fig. 3. Structure of solar collector and related design parameters. 


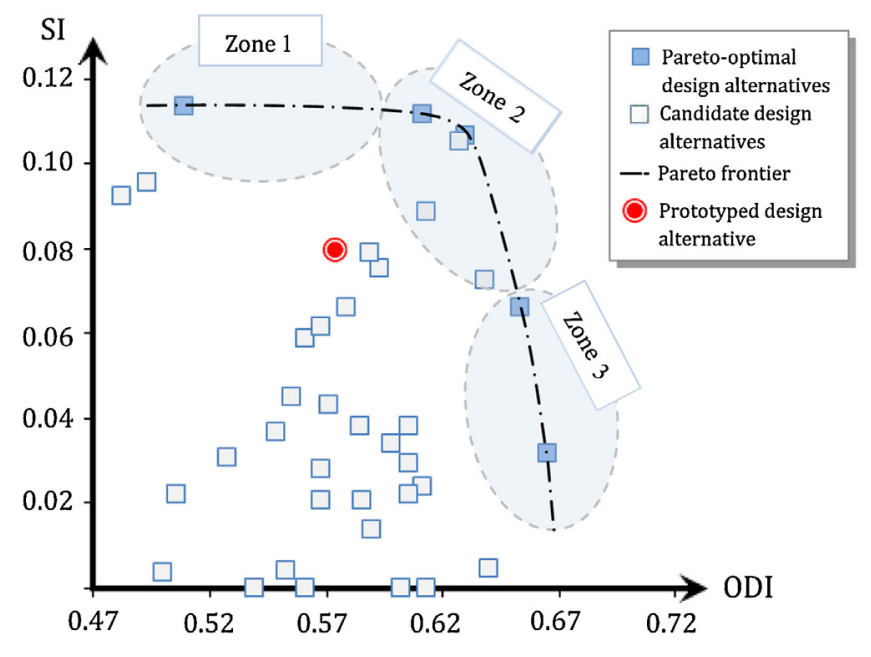

Fig. 4. ODI and SI for each candidate solution for the first scenario.

development tasks needed to this decision. Trade-off must be done between $O D I$ and SI. Many elements are to be taken into account. In this paragraph, general guidelines are derived. In the case where the deadline of validating the product is approaching, decision would prefer a solution in Zone 1 (Fig. 4) since having a feasible solution is a pressing issue in this case. In other circumstances, DM would prefer to explore DA with higher ODI (Zone 2 or Zone 3) even with a low SI. But such a decision implies further investigations and further time to improve SI of chosen DA. These could be done either by improving behaviour models so that the accuracy compared with reference point will better or by realizing other prototypes and other mechanical tests for the selected DA. In our case, we can observe in Fig. 4 that an important gap between $O D I$ of the reference point and the maximum ODI achievable during the second design of experiment. Such a potential of improvement cannot be ignored. Design alternatives with high ODI elicit a low SI which prevents their direct validation. Further development tasks must be done in our case to improve this SI.

Alternative structure that has already been developed and tested in an experimental CSP plant has to be taken into account in reasoning. Its $O D I$ is estimated to 0.53 but its main advantage is its high maturity level. It represents a guarantee for decision makers since it can be directly introduced to the system. In this case, it is more acceptable for the case of truss structure to explore DA with low SI. This scenario is very common in design departments. They are usually encouraged to have a feasible solution with high maturity degree to reduce risk.

In our industrial case, the strategic decision is to adopt a lowcost CSP plant. In the proposed approach, it is expressed by assigning high weight to the objective of structure mass reduction

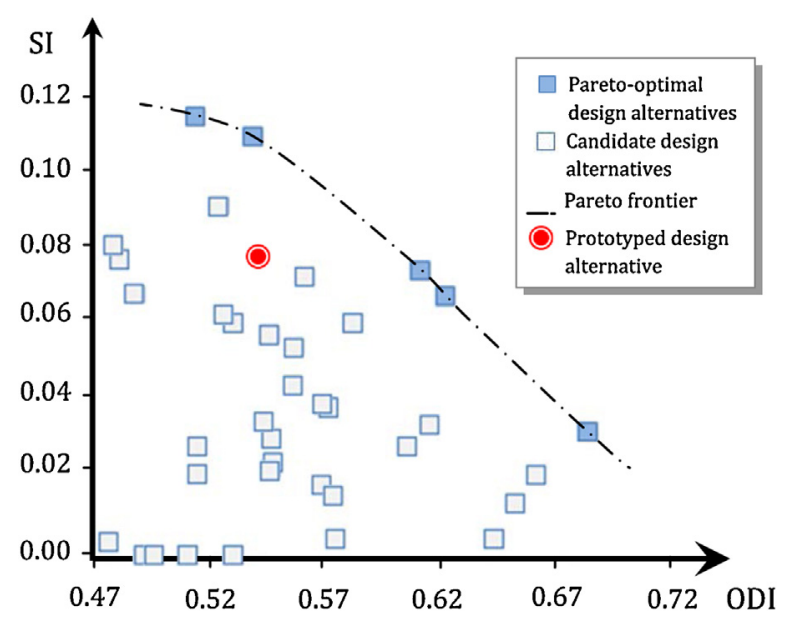

Fig. 5. ODI and SI for each candidate solution for the second scenario. compared with the other design objectives. In many situations, the decision maker wishes to know the effect of giving more importance to some design objectives compared to others. Thereby, we have considered a second scenario in which more importance is given to optical performance (which impacts energy efficiency of the CSP plant) compared to structure mass. Fig. 5 shows the results of $O D I$ and SI calculation for this scenario. It can be seen that the cartography of ODI and SI changed. Such a modification in development strategy can be done at early phases of development process and imply having a holistic view of the global system by trying to understand how individual design decisions impact high-level attributes of the system [16].

\section{Conclusion and future works}

In this work, a multi-criteria decision making model is proposed and tested for the selection of design parameters. It is based on the maximization of the satisfaction of design objectives and minimization of the risk associated with the low accuracy of behaviours models. The construction of proposed indicators passes through the formalization of preferences associated with the achievement of design objectives as well as the decision maker attitude towards risk. The development cost of this multi criteria decision model is counterbalanced by the capitalization on knowledge associated with decision making and the ability to treat a broad spectrum of design alternatives. The strength of proposed accuracy measure (detectability) is to combine on the one hand an objective measure that is the error from reference point and on the other hand a subjective measure that express the degradation of the initial accuracy measure in function of the distance of DP from reference point. This combination allows having an accuracy measure that covers all design space. A future step is to provide thresholds for SI in order to identify a design alternative that can be directly introduced to the system and to treat other case studies.

\section{References}

[1] Zimmer L, Zablit P (2001) Global Aircraft Predesign Based on Constraint Propagation and Interval Analysis. Proceedings of CEAS Conference on Multidisciplinary Aircraft Design and Optimisation, Köln, Germany.

[2] Saridakis K, Dentsoras A (2008) Soft Computing in Engineering Design - A Review. Advanced Engineering Informatics 22(2):202-221.

[3] Krause F-L, Golm F (1996) Planning and Multi-criteria Optimization of Design Processes. Annals of the CIRP 45(1):145-148.

[4] Elwany MH, Khairy AB, Abou-Ali MG, Harraz NA (1997) Combined Multicriteria Approach for Cellular Manufacturing Layout. Annals of the CIRP 46(1):369-372.

[5] Otto KN, Antonsson EK (1993) The Method of Imprecision Compared to Utility Theory for Design Selection Problems. Proceedings of the ASME Design Theory and Methodology, New Mexico, 167-173.

[6] Lemaire M (2014) Mechanic and Uncertainty, Wiley-ISTE. 176p, ISBN: 978-184821-629-7.

[7] Vernat Y, Nadeau J-P, Sebastian P (2010) Formalization and Qualification of models Adapted to Preliminary Design. International Journal on Interactive Design and Manufacturing 4(1):11-24.

[8] Meckesheimer M (2001) A Framework for Metamodel-based Design: Subsystem Metamodel Assessment and Implementation Issues, (PhD thesis) Pennsylvania State University.

[9] Harrington EC (1965) The Desirability Function. Industrial Quality Control 21(10):494-498.

[10] Yager RR (1988) On Ordered Weighted Averaging Aggregation Operators in Multi criteria Decision Making. IEEE Transactions on Systems Man and Cybernetics 18(1):183-190.

[11] Scott MJ, Antonsson EK (2000) Using Indifference Points in Engineering Decisions. Proceedings of the 11th International Conference on Design Theory and Methodology, Baltimore, MD, ASME.

[12] Jin Y, Geslin M, Lu SC-Y (1991) Impact of Argumentative Negotiation on Collaborative Engineering. Annals of the CIRP 56(1):181-184.

[13] Biegel P, Pecht M (1991) Design Trade-offs Made Easy. Concurrent Engineering 1(3):29-40.

[14] El Amine M, Perry N, Pailhès J (2014) Critical Review of Multi-criteria Decision Aid Methods in Conceptual Design Phases: Application to the Development of a Solar Collector Structure. Procedia CIRP 21:497-502.

[15] Kumara V, Shrivastavaa RL, Untawaleb S-P (2015) Fresnel Lens: A Promising Alternative of Reflectors in Concentrated Solar Power. Renewable and Sustainable Energy Reviews 4:376-390.

[16] Lu SC-Y, Elmaraghy W, Schuh G, Wilhelm R (2007) A Scientific Foundation of Collaborative Engineering. Annals of CIRP 56(2):605-634. 